\title{
Role of Ultrasound as Compared with ERCP in Patient With Obstructive Jaundice
}

\author{
Karki $\mathrm{S},{ }^{1}$ Joshi KS, ${ }^{1}$ Regmi $S,{ }^{1}$ Gurung RB, ${ }^{2}$ Malla $\mathrm{B}^{3}$
}

\author{
${ }^{1}$ Department of Radiodiagnosis and Imaging \\ ${ }^{2}$ Department of Internal medicine \\ ${ }^{3}$ Department of Surgery \\ Dhulikhel Hospital- Kathmandu University Hospital \\ Kathmandu University School of medical Science \\ Dhulikhel Hospital, Kavre, Nepal
}

\section{Corresponding Author}

Subindra Karki

Department of Radiodiagnosis and Imaging

Dhulikhel Hospital- Kathmandu University Hospital Kathmandu University School of medical Science

Dhulikhel, Kavre, Nepal

E-Mail: subindrakarki@gmail.com

\section{Citation}

Karki S, Joshi KS, Regmi S, Gurung RB, Malla B. Role of Ultrasound as Compared with ERCP in Patient with Obstructive Jaundice. Kathmandu Univ Med J 2013;43(3):237-240.

\begin{abstract}
Background

The diagnosis of obstructive jaundice relies on proper history taking, clinical examination, laboratory investigations and different non invasive imaging modalities like Ultrasonography (USG), Cholangio Computed Tomography (CCT), Magnetic resonance Imaging (MRI) with Magnetic Resonance Cholangio Pancreatography (MRCP) and invasive modalities like endoscopic retrograde cholangiography (ERCP) and percutaneous trans hepatic cholangiography (PTC).
\end{abstract}

\section{Objective}

To compare the role of ultrasound with endoscopic retrograde cholangiography and to determine the major causes of obstructive jaundice in our prospect.

\section{Methods}

This was a prospective, analytical study conducted on 88 patients presenting to Department of Radiodiagnosis and Imaging at Dhulikhel Hospital-Kathmandu University hospital from March 2011 to August 2012 with clinical diagnosis of obstructive jaundice. Sonographic evaluation was performed in Siemens acusion $x-150$ and $x-300$. The final diagnosis was made by endoscopic retrograde cholangiography and /or surgery and confirmed histopathologically.

\section{Results}

The most common benign causes of obstructive jaundice were choledocholithiasis (63\%), CBD stricture (12.3\%), cholangitis (8\%) and pancreatitis $(6.85 \%)$ whereas cholangio carcinoma (6.85\%) and carcinoma head of pancreas (4\%) comprised of the malignant causes. Ultrasonography had sensitivity of $100 \%$ and specificity of $89 \%$ in detecting choledocholithiasis. It was found to be $98.78 \%$ sensitive and $83.33 \%$ specific in cholangiocarcinoma. Similarly in pancreatitis, the sensitivity of ultrasonography was $97.59 \%$ and sensitivity was $66.67 \%$.

\section{Conclusion}

Ultrasonography acts as a valuable diagnostic imaging modality in detecting the causes of obstructive jaundice. Due to its easy availability, non invasive nature and cost effectiveness, it can be considered as the first line imaging technique/ tool. ERCP is the invasive imaging tool and can be used for both diagnostic and therapeutic purpose.

\section{KEY WORDS}

$E R C P$, obstructive jaundice, ultrasonography 


\section{INTRODUCTION}

Obstructive jaundice is a common surgical problem that occurs when there is an obstruction to the passage of bilirubin from liver to the intestine. ${ }^{1}$ The importance of differentiating obstructive jaundice from non obstructive jaundice lies in the fact that there is a great difference in the existing treatment between the two. Recent years have witnessed a rapid and continuous evolution in the diagnosis of biliary obstructive disease. ${ }^{2}$ Commonly used procedures include Ultrasonography(USG), Computed Tomography (CT), Endoscopic retrograde cholangiopancreatography (ERCP), Percutaneous transhepatic cholangiography (PTC) and Magnetic resonance cholangiopancreatography (MRCP). ${ }^{3}$

Invasive modalities such as PTC and ERCP are mainly dependent on personal skills of the operator and are associated with relatively high rate of complications. Even though many new imaging modalities are available, because of the cost effectiveness, easy availability and non invasive nature USG can be considered as first line imaging tool.

\section{METHOD}

This was a prospective, analytical study conducted on 88 patients presented to Department of Radiodiagnosis and Imaging at Dhulikhel Hospital-Kathmandu University hospital from March 2011 to August 2012 with clinical diagnosis of obstructive jaundice.

Patients with clinical jaundice (symptomatic and asymptomatic), deranged liver function test (raised total, direct bilirubin and Alkaline phosphatase) were included in the study. Immediate post ERCP cases were excluded from the study. The study was approved by Institutional Review Committee of Kathmandu University School of Medical Sciences. A detailed history was taken and written informed consent was taken and proforma was filled up. The patients were evaluated clinically to detect the presence of jaundice and biochemical assessment for serum bilirubin, alkaline phosphatase and other relevant pathological tests were done. Sonographic evaluation was performed in Siemens acusion $x-150$ and $x-300$ USG machine using 3.5MHz convex transducer in supine and lateral decubitus position in all patients preferably on an overnight fasting state. Careful scanning of the entire course and caliber of the duct system whenever possible was done to trace the extent of the duct dilatation and to localize the level of obstruction. In several cases $5 \mathrm{MHz}$ linear transducer was used and water was given orally to provide better acoustic window to image distal common bile duct and pancreas. Gray scale B mode study was performed transabdominally using axial, subcostal and intercostal approach. The final diagnosis was made by ERCP and /or CT, surgery and confirmed histopathologically. Statistical analysis was done by using SPSS software version 16.0.Sensitivity, specificity, positive predictive value (PPV); negative predictive values (NPV) were also calculated.

\section{RESULT}

Out of total 88 patients $29(33 \%)$ were male and 59(67\%) were female. The age of patients ranged from 5 to 82 years (mean age 50.27 years). Table 1 shows the frequency and percentage of the symptoms.

Table 1. Frequency and percentage of presenting symptoms.

\begin{tabular}{|lcc|}
\hline Chief complaint & Frequency & Percent \\
\hline Nausea & 76 & 86.4 \\
\hline Vomiting & 17 & 19.3 \\
\hline Pain & 75 & 85.2 \\
\hline Weight loss & 6 & 6.8 \\
\hline Loss of appetite & 14 & 15.9 \\
\hline Itching & 9 & 10.2 \\
\hline Jaundice & 34 & 38.6 \\
\hline Fever & 10 & 11.4 \\
\hline
\end{tabular}

The sonological findings encountered in patient with obstructive jaundice was dilatation of intrahepatic biliary radicals.USG was found to be $94.8 \%$ sensitive and $100 \%$ specific.

The most common benign causes of obstructive jaundice were choledocholithiasis(63\%), CBD stricture(12.3\%), pancreatitis(6.85\%) and cholangitis(8\%), whereas cholangiocarcinoma(6.85\%) and carcinoma head of pancreas(4\%) accounted for the malignant causes.

Some rare causes of jaundice like Choledochal cyst (2.74\%), gall bladder malignancy with hilar nodes $(1.37 \%)$, groove pancreatitis (1.37\%), Caroli's disease $(1.37 \%)$, intrahepatic biliary radical parasite (Ascaris lumbricoides) (1.37\%), duodenitis (1.37\%), periampullary carcinoma (1.37\%) were also encountered during our study.

USG has sensitivity of $100 \%$ and specificity of $89 \%$ in detecting choledocholithiasis. Similarly, USG was found to be $98.78 \%$ sensitive and $83.33 \%$ specific in cases of cholangiocarcinoma and $97.59 \%$ sensitive and $66.67 \%$ specific in pancreatitis. Ultrasound was found to have very high accuracy in detecting biliary tract dilatation with sensitivity of $94.8 \%$ and specificity of $100 \%$. However the detection of CBD stricture by USG was not statically significant as compared to ERCP.

The agreement between USG and ERCP findings in the Table 2. Comparison between the findings of Ultrasound and ERCP.

\begin{tabular}{|cccccc|}
\hline USG & Frequency & Percent & ERCP & Frequency & Percent \\
\hline IHBRD & 55 & 62.5 & IHBRD & 58 & 65.9 \\
\hline EDC & 44 & 60.3 & EDC & 46 & 63 \\
\hline EDS & 5 & 6.8 & EDS & 9 & 12.3 \\
\hline EDG & 3 & 4.1 & EDG & 0 & 0 \\
\hline
\end{tabular}

Note IHBRD- intrahepatic biliary radical dilatation,ED-extrahepatic duct, C-calculus, S-stricture, G-growth, GB-gallbladder. 
patients with clinical diagnosis of obstructive jaundice are shown in Table 2.

\section{DISCUSSION}

When evaluating a case of obstructive jaundice, the aim of the radiologist is to confirm the presence of obstruction and to identify its location, extent and probable cause. The success rate in diagnosing specific cause has continued to improve with advancing high resolution equipment, scanning technique and interpretive skills. With the availability of USG, CCT, MRI, including MRCP, ERCP and PTC, diagnostic approach in a patient with biliary tract pathology has been completely revolutionized with accuracy of radiological diagnosis approaching $98 \%$ when combined with clinical data. ${ }^{4}$

USG has always been used as the initial screening procedure because of the various advantages like easy availability, cost effectiveness, no requirement of contrast material and lack of ionizing radiation. However, although it is well suited to visualize the Common Hepatic Duct (CHD) and proximal $C B D$, one of its major limitations is assessment of the distal $\mathrm{CBD}$ and pancreas, which are often obscured by overlying bowel gas in about $30-50 \%$ of the patients. ${ }^{5,6}$

In our study, the major causes of obstructive jaundice were choledocholithiasis (fig 1, 2) followed by CBD stricture(fig 3), cholangitis, cholangio carcinoma, and carcinoma head of pancreas which is comparable with previous study. ${ }^{7}$

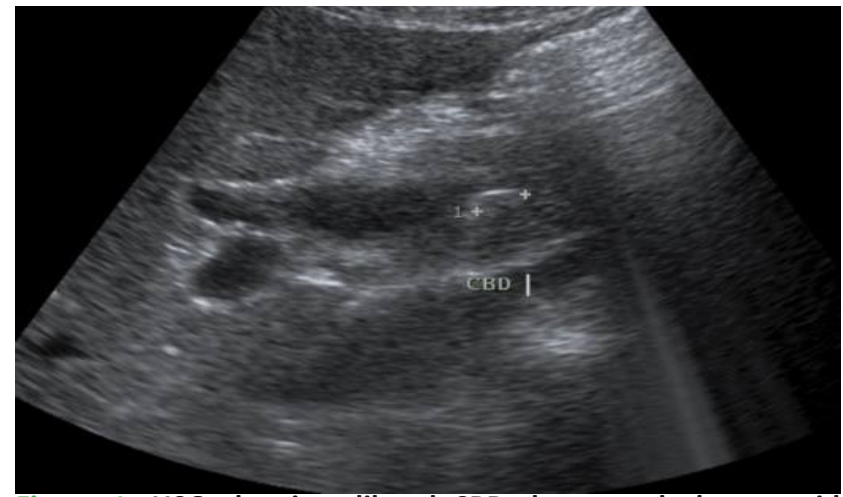

Figure 1. USG showing dilated CBD due to calculus at mid segment of CBD.

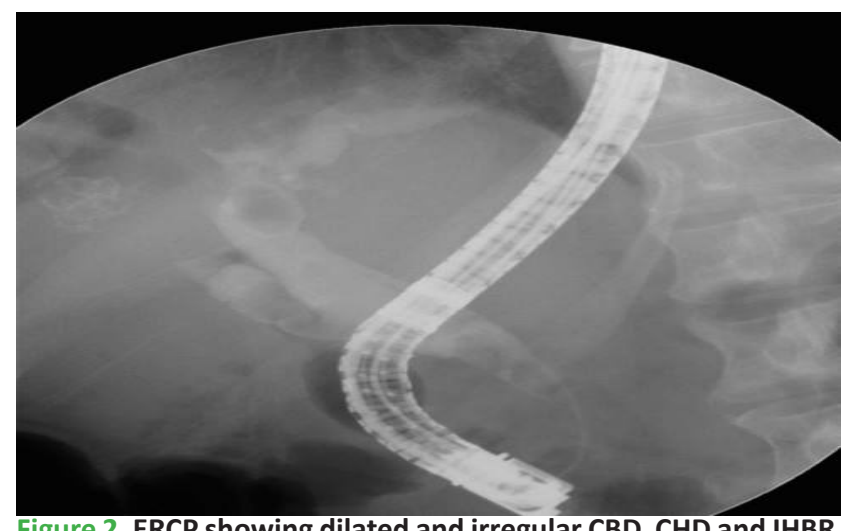

Figure 2. ERCP showing dilated and irregular CBD, CHD and IHBR dilatation with multiple CBD calculi.

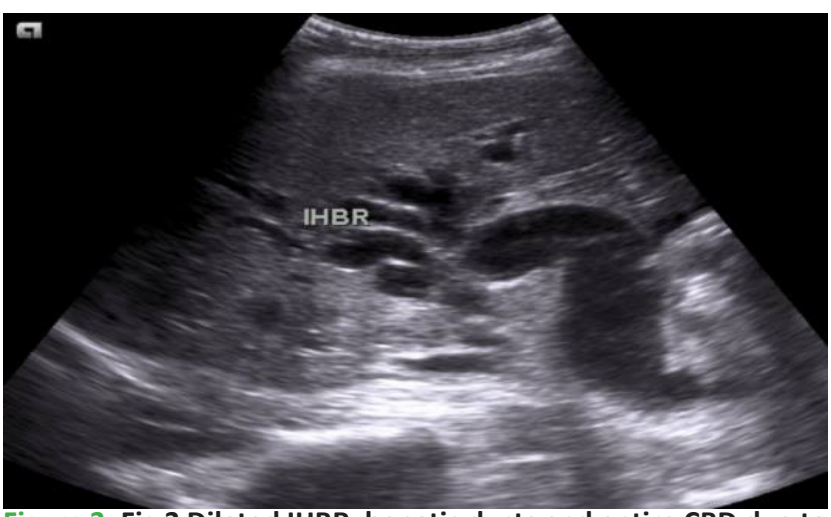

Figure 3. Fig 3 Dilated IHBR, hepatic ducts and entire CBD due to distal CBD stricture.

Several studies have shown that ultrasonography has very high sensitivity of upto $97 \%$ in diagnosing the cause of obstructive jaundice. The success rate in diagnosing specific cause has continued to improve with advancing high resolution equipment, scanning technique and interpretive skills. ${ }^{8}$

In a study conducted by Varghese et al, ultrasound was found to have specificity of $100 \%$ and diagnostic accuracy of $89 \%$ respectively, in diagnosing choledocholithiasis. ${ }^{9}$

In the present study, we found that sensitivity and specificity of USG in diagnosing choledocholithiasis was $100 \%$ and $89 \%$ respectively which were comparable to that of some series $97 \%$ of 150 patients, $95 \%$ of 40 patients and $86 \%$ of 35 patients. ${ }^{6,10,11}$

Similarly in a study performed by Upadhyaya et al, USG was able to identify the level in $83.50 \%$ and cause in $77 \%$ cases of obstructive jaundice. ${ }^{3}$

In literature, a sensitivity range of 20 to $80 \%$ is documented when ultrasonography is used to detect CBD stones. ${ }^{12}$ These considerable differences in sensitivity among various case series are partially attributable to the impossibility of approaching the distal CBD and ampullary region in obese patients, as well as to the variability of the ultrasonography technique applied. The studies with poor results are mostly older studies when the resolution of ultrasound machine was not good and it was a new technique still being learnt.

The advent of ERCP dramatically changed the diagnosis and treatment of biliary and pancreatic diseases. It currently remains the standard of reference for imaging the bile duct and the pancreatic duct.

In our study, ERCP was found to be the much sensitive in detecting CBD stricture as compared to USG which was similar to the study performed by Upadhyaya et.al. ${ }^{3}$

PTC is particularly useful when proximal pathology is suspected based on previous screening procedures. A major drawback of these procedures is their invasive nature which may result in complications. Several authors have reported that ERCP is associated with a high rate of morbidity (1-7\%). ${ }^{13-17}$ 
The recent studies have demonstrated that ERCP is not an innocuous procedure. Its morbidity rate ranges between $5 \%$ and $19 \%$ while diagnostic ERCP has a mortality rate ranging from $0.5 \%$ to $2 \%{ }^{18,19}$

This mortality rate is doubled in therapeutic ERCP, which obliges physicians not to use it diagnostically, but rather to use it therapeutically only when there is already a certain diagnosis. ${ }^{20}$

MRI with MRCP, CCT is the newer non invasive imaging modalities with very high accuracy in detecting the cause and level of obstruction. However due to cost effectiveness, easy availability USG is considered as first choice option in diagnosing biliary tract disease. If the suspicion posed by clinical and laboratory findings is not confirmed at US, the diagnosis must be achieved with the aid of higher imaging modalities like MRCP/CCT, so as to select candidates for therapeutic ERCP, PTC or surgery. ${ }^{2}$

\section{REFERENCES}

1. Mohamed S, Syed Al. Management of Obstructive Jaundice: Experience in a tertiary care surgical unit. Pakistan Journal of Surgery 2007, 23:23-25

2. Ferrari FS, Fantozzi F, Tasciotti L, Vigni F, Scotto F, Frasci P. US, MRCP, CCT and ERCP: a comparative study in 131 patients with suspected biliary obstruction. Med Sci Monit. 2005;11(3):8-18.

3. Upadhyaya V, Upadhyaya DN, Ansari MA.Comparative assessment of imaging modalities in biliary obstruction. Indian J Radiol Imaging 2006;16:577-82.

4. Fulcher AS, Turner MA. MR Cholangiopancreatography. RCNA. 2002; 40: 1363-76.

5. Ferruci JT, Jr. Body Ultrasonography. N Eng/ J Med. 1979;300:590-602.

6. Taylor KJW, Rosenfield AT, de Graaff CS. Anatomy and pathology of the biliary tree as demonstrated by ultrasound. Clinics in Diagnostic Ultrasound.1979; 1:103-21.

7. Kiani AA,Javaid RH,Ghaffar A, Khan S. Ultrasonography in obstructive jaundice. Professional Med J. July-Aug 2012;19(4): 436-441.

8. Haubeck C, Pedersen J, Burcarth FJG.Dynamic sonography in evaluation of jaundice. Amer J Radio. 1981; 123: 1071-9.

9. Varghese JC, Liddell RP, Farrell MA. Diagnostic accuracy of magnetic resonance cholangiopancreatography and ultrasound compared with direct cholangiography in the detection of choledocholithiasis. Clin Radiol. 2000; 55(1):25-35

10. Isikoff MB,Diaconis JN .Ultrasound: A New Diagnostic Approach to the Jaundiced Patient. JAMA. 1977;238(3):221-223.

\section{CONCLUSION}

With development of high resolution ultrasound machine, better preparation and positioning of patients and improved techniques, ultrasound has proven to be highly accurate in diagnosing the cause of obstructive jaundice and can be considered as the first line investigation. Considering the risks of invasive modalities including ERCP and PTC, these modalities could be indicated where therapeutic interventions are planned.

\section{ACKNOWLEDGEMENT}

Authors would like to thank Mr Seshananda Sanjel from Department of Community medicine for helping in statistical analysis.

11. Malini S, Sabel J.Ultrasonography in obstructive jaundice. Radiology. 1977 May;123(2):429-33.

12. Vashisht $S$, Thulkar $S$. Imaging of obstructive biliopathy. In: Berry M, Chowdhury V, Mukhopadhyay S, Suri S.Diagnostic radiology gastrointestinal and hepato-biliary imaging. 2nd ed. New Delhi: Jaypee brothers medical publishers; 2004: 288-313.

13. Bilbao MK, Dotter CT, Lee TG, Katon RM. Complications of endoscopic retrograde cholangiopancreatography. A study of 10,000 cases. Gastroenterology. 1976; 70: 314-20.

14. Hamilton I, Lintott DJ, Rothwell J, Axon ATR. Acute pancreatitis following Endoscopic retrograde cholangiopancreatography. Clin Radiol. 1983; 34: 543-6.

15. Thoeni RF, Fel SC, Goldberg HI.CT detection of asymptomatic pancreatitis following ERCP. Gastrointest Radiol. 1990; 15: 291-5.

16. Lo SK, Chen J. The role of ERCP in choledocholithiasis. Abdom Imaging. 1996 Mar-Apr;21(2):120-32.

17. Masci E, Toti G, Mariani A, Curioni S, Lomazzi A, Dinelli M,et al. Complications of diagnostic and therapeutic ERCP:A prospective multicenter study. Am J Gastroenterol. 2001 Feb;96(2):417-23.

18. Shaffer EA. Gallstone disease: Epidemiology of gallbladder stone disease. Best Pract Res Clin Gastroenterol. 2006;20(6): 981-996.

19. Attasaranya S, Fogel EL, Lehman GA. Choledocholithiasis,ascending cholangitis, and gallstone pancreatitis. Med Clin North Am. 2008; 92(4): 925-960.

20. Mallery JS, Baron TH, Dominitz JA, Goldstein JL,Hirota WK, Jacobson BC, et al. Complications of ERCP. Gastrointest Endosc. 2003; 57(6): 633-638. 